\title{
A flaring megamaser in Mrk 348
}

\author{
Alison B. Peck, Heino Falcke, Christian Henkel, Karl M. Menten, \\ Yoshiaki Hagiwara \\ MPIfR, Auf dem Hügel 69, D-53121 Bonn, Germany \\ Jack F. Gallimore \\ Bucknell Univ., Lewisburg, PA 17837
}

James S. Ulvestad

NRAO, P.O. Box 0, Socorro, NM 87801

\begin{abstract}
We report new observations of the $\mathrm{H}_{2} \mathrm{O}$ megamaser in the Seyfert 2 galaxy Mrk 348. Following our initial detection in 2000 March using the Effelsberg $100 \mathrm{~m}$ telescope, a re-analysis of previous data on this source indicates that the maser was present but only marginally detectable in late 1997. Monitoring through late 2000 shows that the maser has again decreased to its original level. The $\mathrm{H}_{2} \mathrm{O}$ line is redshifted by $\sim 130 \mathrm{~km} \mathrm{~s}^{-1}$ with respect to the systemic velocity, is extremely broad, with a FWHM of $130 \mathrm{~km} \mathrm{~s}^{-1}$, and has no detectable high velocity components within $1500 \mathrm{~km} \mathrm{~s}^{-1}$ on either side of the strong line. Followup VLBA observations show that the maser emission emanates entirely from a region $\leq 0.25 \mathrm{pc}$ in extent, toward the base of the radio jet.
\end{abstract}

\section{Introduction}

Mrk 348 (NGC 262) is a Seyfert 2 galaxy at a redshift of 0.01503 (Huchra et al. 1999). The galaxy is classified as an S0 with a low inclination, and exhibits a large $\mathrm{HI}$ halo which may have been produced by an interaction with the companion galaxy NGC 266 (e.g. Simkin et al. 1987). VLBA images (Ulvestad et al. 1999) reveal a small-scale double continuum source, the axis of which is aligned with the optical ([OIII], Capetti et al. 1996) emission. Astrometry indicates that the optical and VLBI cores are nearly coincident. Apparent subrelativistic expansion of the inner two jet components has been detected by Ulvestad et al. (1999). Ground-based observations (Simpson et al. 1996) show evidence of a dust lane crossing the nucleus and an ionization cone. Attempts to detect the expected obscuring torus at radio wavelengths (e.g. Hi, Gallimore et al 1999; CO, Taniguchi et al. 1990; free-free absorption, Barvainis \& Lonsdale 1998) have not been successful.

The compact radio source in Mrk 348 is unique among Seyferts in that it is very bright and extremely variable. The observations presented here were made 


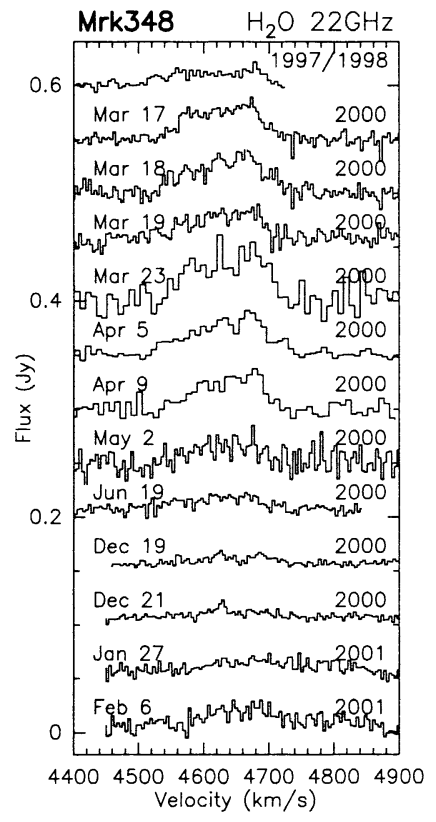

Figure 1. Single dish profiles from Effelsberg $100 \mathrm{~m}$ telescope. The peak flux in the line was $\sim 40 \mathrm{mJy}$ on April 9, but decreased to $18 \mathrm{mJy}$ by June 19 .

during a local minimum, when the total continuum flux density at $22 \mathrm{GHz}$ was $\sim 0.6 \mathrm{Jy}$.

\section{Observations}

The initial detection of the flaring maser in Mrk 348 using the Effelsberg $100 \mathrm{~m}$ telescope took place in 2000 March. Re-analysis of previous data on this source (top profile, Fig. 1) indicates that the maser was also present but only marginally detectable in late 1997. Monitoring through June 2000 showed that the maser again decreased to its original level within 2 months, as shown in Fig. 1. The June 19 profile indicates a peak flux of $\sim 18 \mathrm{mJy}$. Resumption of the monitoring program in December 2000 showed little change in the line flux, though February 2001 observations yield a slight strengthening of the line. We will continue to monitor this source and additional follow-up observations will be attempted if it is seen to flare again.

The $\mathrm{H}_{2} \mathrm{O}$ maser line in Mrk 348 is extremely broad, with a FWHM of $\sim 130 \mathrm{~km} \mathrm{~s}^{-1}$, though in many of the monitoring epochs the emission appears to consist of 2 lines which can be fit by Gaussian functions with FWHM of $\sim 60$ $\mathrm{km} \mathrm{s}^{-1}$ and $\sim 100 \mathrm{~km} \mathrm{~s}^{-1}$ respectively, separated by $\sim 70 \mathrm{~km} \mathrm{~s}^{-1}$. There are no detectable high velocity components within $1500 \mathrm{~km} \mathrm{~s}^{-1}$ on either side of the strong emission line.

The VLBA observations took place on 2000 June 10. March Effelsberg observations indicated that the line was too broad to fit in a single $16 \mathrm{MHz}$ 


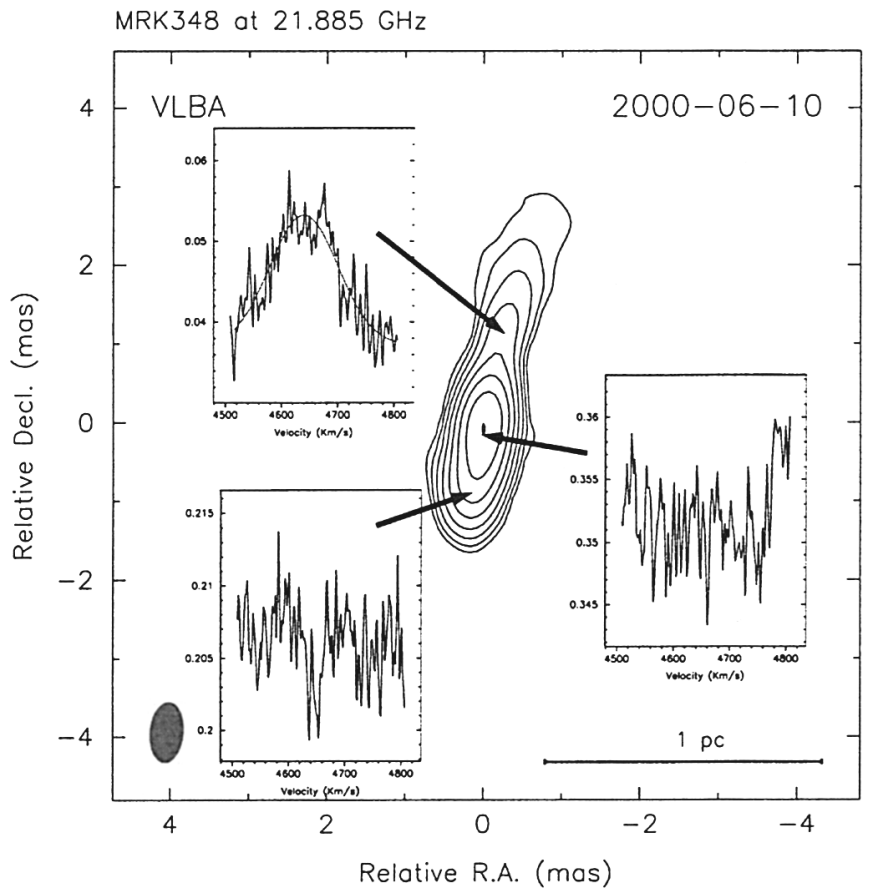

Figure 2. Line profiles from VLBI data. The continuum map is naturally weighted. The lowest contour is $3 \mathrm{mJy}$ and the peak is 352 mJy. The RMS noise in the continuum map is $<1 \mathrm{mJy} / \mathrm{beam}$, and in the line profiles is $\sim 4 \mathrm{mJy} /$ beam/channel.

IF (FWZP $>250 \mathrm{~km} \mathrm{~s}^{-1}$ ), so 2 IFs of $16 \mathrm{MHz}$ each were used, overlapped by $5 \mathrm{MHz}$. Following calibration, the overlapping channels were removed and the 2 IFs were added together to yield a single cube of 174 channels covering 23 $\mathrm{MHz}$. Line profiles of the resulting cube are shown in Fig. 2, superimposed on a continuum map made from 20 line-free channels. The maser emission is clearly seen to lie along the line of sight to the jet, rather than the core which is thought to lie toward the southern end of the continuum source. The Gaussian fit to the line shown in the first profile has an amplitude of $16 \pm 2 \mathrm{mJy}$ and an integrated flux of $2.4 \pm 0.3 \mathrm{Jy} / \mathrm{beam} / \mathrm{km} \mathrm{s}^{-1}$, indicating that all of the flux measured in the Effelsberg 19 June observation has been recovered. The FWHM is $142 \pm 9 \mathrm{~km} \mathrm{~s}^{-1}$ centered on $\mathrm{V}_{\mathrm{LSR}}=4640 \pm 2 \mathrm{~km} \mathrm{~s}^{-1}$, consistent with the single-dish measurements and redshifted by $131 \mathrm{~km} \mathrm{~s}^{-1}$ with respect to the systemic velocity. A tentative 2 component fit to the data yields a narrower line at $4683 \mathrm{~km} \mathrm{~s}^{-1}$ with FWHM $\sim 60 \mathrm{~km} \mathrm{~s}^{-1}$ and amplitude $\sim 8 \mathrm{mJy}$, and a broader line at $4620 \mathrm{~km} \mathrm{~s}^{-1}$ with FWHM $\sim 100 \mathrm{~km} \mathrm{~s}^{-1}$ and amplitude $\sim 12 \mathrm{mJy}$, again consistent with our singledish measurements. No maser emission is seen toward any other region of the radio source, only toward the jet, and here the maser emission is unresolved at our angular resolution of $0.42 \times 0.76$ mas. This corresponds to a linear size of less than $0.25 \mathrm{pc}$ (assuming $\mathrm{H}_{0}=75 \mathrm{~km} \mathrm{~s}^{-1} \mathrm{Mpc}^{-1}$ ). 


\section{Conclusions}

During early 2000, the $\mathrm{H}_{2} \mathrm{O}$ emission toward Mrk 348 showed a dramatic intensity increase which coincided with a significant increase in the flux of the nuclear radio continuum source. The unusual line profile leads us to suspect that this source, and possibly NGC 1052, might belong to a class of megamaser galaxies in which the amplified emission is the result of an interaction between the radio jet and an encroaching molecular cloud, rather than occurring in a circumnuclear disk. Analysis of our recent VLBA observations indicates that the emission does indeed arise along the line of sight to the jet in Mrk 348 (see Fig. 2), confirming this prediction. The very high linewidth occurring on such small spatial scales indicates that the $\mathrm{H}_{2} \mathrm{O}$ emission arises from a shocked region at the interface between the energetic jet material and the molecular gas in the cloud where the jet is boring through. This hypothesis is supported by the spectral evolution of the continuum source (Brunthaler, priv. comm.), which showed an inverted radio spectrum with a peak at $22 \mathrm{GHz}$, later shifting to lower frequencies. By analogy to IIIZw2 (Brunthaler et al. 2000) this would indicate the formation and evolution of very compact hotspots propagating through a dense medium. In this scenario, the recent high frequency radio continuum flare and the northward movement of the brightest continuum component are attributable to the impact between the jet and the molecular cloud. The very close temporal correlation between the flaring activity in the maser emission and the continuum flare further suggest that the masing region and the continuum hotspot are nearly coincident and may be different manifestations of the same dynamical events.

Acknowledgments. We would like to thank Barry Clark and the staff at NRAO for promptly scheduling and correlating the VLBA observations as a Target of Opportunity experiment. The National Radio Astronomy Observatory is a facility of the National Science Foundation operated under a cooperative agreement by Associated Universities, Inc.

\section{References}

Barvainis, R. \& Lonsdale, C. 1998, AJ, 115, 885

Brunthaler, A., Falcke, H., Bower, G. C., Aller, M. F., Aller, H. D.,Teräsranta, H., Lobanov, A. P., Krichbaum, T. P. \& Patnaik, A. R. 2000, A\&A, 357, L45

Capetti, A., Axon, D. J., Macchetto, F., Sparks, W. B. \& Boksenberg, A. 1996, ApJ, 469,554

Falcke, H. Henkel, C., Peck, A. B., Hagiwara, Y., Prieto, M. A. \& Gallimore, J. F. 2000, A\&A, 358, L17

Gallimore, J. F., Baum, S. A., O'Dea C. P., Pedlar, A. \& Brinks, E. 1999, ApJ, 524, 684

Huchra, J. P., Vogeley, M. S. \& Geller, M. J. 1999, ApJS, 121, 287

Simkin, S. M, Su, H.-J., van Gorkom, J. \& Hibbard, J. 1987, Science, 235, 1367

Simpson, C., Mulchaey, J. S., Wilson, A. S., Ward, M. J. \& Alonso-Herrero, A. 1996, ApJ, 457, L19

Taniguchi, Y., Kameya, O., Nakai, N. \& Kawara, N. 1990, ApJ, 358, 132

Ulvestad, J. S., Wrobel, J. M., Roy, A. L., Wilson, A. S., Falcke, H \& Krichbaum, T. P. 1999, ApJ, 517, L81 